\title{
Numerical Analysis of Electrogastrogram in the Elderly
}

\author{
Hiroki Takada, Yasuyuki Matsuura, and Masaru Miyao
}

\begin{abstract}
An electrogastrogram (EGG) is a recording of the electric activity of the stomach as measured on the abdominal surface. In this study, our goal was to obtain a mathematical model of an EGG in the elderly. Subjects included 7 males and 7 females ranging from 65 to 76 years in age. Initially, we applied the Wayland algorithm to the EGG in order to measure the degree of determinism. However, we could not determine whether the EGG could be generated by a chaotic process. Incidentally, the waveform of the electric potential in the interstitial cells of Cajal is similar to the graphs generated from the numerical solutions of the Van der Pol equation. We therefore added the Van der Pol equation to a periodic function and used random white noise to represent the intestinal motility and other biosignals. We then compared and evaluated the EGG and numerical solutions on the basis of the translation error in the Wayland algorithm and the maximum Lyapunov exponent in Rosenstein's algorithm. By projecting the data from stationary EGGs and the numerical solutions onto the $E_{\text {trans }}-\lambda$ plane, we could qualitatively evaluate the affinity between them. In conclusion, a mathematical model of the EGG in the elderly includes stochastic differential equations that do not describe the stochastic resonance (SR). Conversely, the SR is statistically applicable to the mathematical model of the stationary EGG in the healthy young. In this paper, we also discuss the physical meaning of the mathematical model of EGGs in the elderly.
\end{abstract}

Index Terms-Electrogastrography, numerical solution, wayland algorithm, lyapunov exponent, stochastic resonance (SR).

\section{INTRODUCTION}

Percutaneous electrogastrography is a useful method for examining human gastric electrical activity without invasion. Human gastric electrical activity cannot be measured by other methods such as magnetic resonance imaging (MRI) or gastro-fiberscopy. An electrogastrogram (EGG) is evaluated by comparing the values of mean frequency and power obtained with values derived from the spectrum analysis of previous EGG studies. However, the amount of information that can be obtained from this comparison is limited. Therefore, EGGs are used less often than electrocardiograms, electroencephalogram, and other biosignal measurements.

Manuscript received September 20, 2012; revised October 23, 2012. This work was supported in part by the Ministry of Education, Culture, Sports, Science and Technology (MEXT, Japan), a Grant-in-Aid for JSPS Fellows, and the Hori Information Science Promotion Foundation.

H. Takada is with the Graduate School of Engineering, University of Fukui, 3-9-1 Bunkyo Fukui-City, Fukui 910-8507, Japan (e-mail: takada@u-fukui.ac.jp).

Y. Matsuura is with the Headquarters for Innovative Society-Academia Cooperation, University of Fukui, 3-9-1 Bunkyo Fukui-City, Fukui 910-8507, Japan (phone: +81-776-27-8795; fax: +81-776-27-8420; e-mail: matuura@u-fukui,ac.jp)

M. Miyao is with the Graduate School of Information Science, Nagoya University, Furo-cho Chikusa-ku Nagoya, Aichi 464-8603, Japan (e-mail: mmiyao@is.nagoya-u.ac.jp).
However, using a mathematical model for an EGG makes it possible to obtain additional information.

In 1921, Alvarez reported performing EGG for the first time in humans [1]. In an EGG, the electrical activity of the stomach is recorded through electrodes placed on the surface of the abdominal wall. In the stomach, a pacemaker located on the side of the greater curvature generates electrical activity at a rate of 3 cycles per minute $(3 \mathrm{cpm})$. The electrical signal is then transferred to the pyloric side [2].

A gastric electrical potential is generated by the interstitial cells of Cajal (ICCs) [3]. ICCs are pacemaker cells that spontaneously depolarize and repolarize at a rate of $3 \mathrm{cpm}$.

The waveform of the electric potential in ICCs is similar to the graphs of the numerical solutions of the Van der Pol equation. We thus added the Van der Pol equation to a periodic function and used random white noise to represent intestinal motility and other biosignals.

$$
\begin{gathered}
\dot{x}=y-\alpha \cdot \operatorname{grad} f(x)+s(t)+\mu w_{1}(t) \\
\dot{y}=-x+\mu w_{2}(t)
\end{gathered}
$$

The function $s(t)=\sin \omega t$ and white noise $w i(t)$ represent the weak and random intestinal movements and other biosignals $(i=1,2)$, respectively. The double-well potential, $f(x)$, generates depolarization and repolarization in ICCs.

In this study, the gastrointestinal motility was measured with the purpose of obtaining a mathematical model of an EGG. The model along with speculating factors was used to describe the diseases resulting from constipation and erosive gastritis.

Previous studies have discussed solutions to the forward and inverse problems associated with the dynamics of generating the gastric electrical potential. These studies advocate the use of current dipoles in an ellipsoid and computer simulations to generate a mathematical model for the stomach. However, available results of nonlinear analyses of the EGG are insufficient. In order to examine whether or not a mathematical model accurately describes EGG data, we have proposed a projection of a time series on a two-dimensional plane, $E_{\text {trans }}-\lambda$, estimated by using the Wayland and Rosenstein algorithms [4].

The Wayland algorithm has been developed in order to evaluate the degree of determinism for dynamics that generate a time series [5]. This algorithm can estimate a parameter called the translation error $\left(E_{\text {trans }}\right)$ to measure the smoothness of flow in an attractor, which is assumed to generate the time-series data.

Chaos processes generate complexity in an attractor, which can be reconstructed from a time series [6]. These processes have a sensitive dependence on the initial conditions and can be quantified using the Lyapunov exponent [7], [8]. If the Lyapunov exponent has a positive value, the dynamics are 
considered to be a chaos process. In this study, Rosenstein's algorithm [7], [8] was used to calculate the maximum Lyapunov exponent (MLE), $\lambda$.

According to the analysis of the degree of determinism for the time series dynamics, the EGG data obtained $30 \mathrm{~min}$ after a subject's postural change were significantly different from the initial EGG data [4]. The initial EGG data were also regarded as a stationary time series in this study. During the latter period, the autonomic nervous system could be represented by a stationary process because it controls the gastric electrical activity, as measured by an EGG.

In this study, the gastrointestinal motility was measured with the purpose of obtaining a mathematical model of the stationary EGG. In addition, we examined whether numerical solutions to stochastic resonance (SR) [9] would fit the EGG data.

\section{MATERIAL AND METHOD}

The subjects were 14 healthy people $(7$ males and 7 females) aged between 65 and 76 years. A sufficient explanation of the experiment was provided to all the subjects, and a written consent was obtained from them.

\section{A. Experimental Procedure}

EGGs were obtained at $1 \mathrm{kHz}$ for $30 \mathrm{~min}$ by using an A/D converter [AD16-16U (PCI) EH; CONTEC, Japan] with a subject in the sitting position. The EGGs were amplified using a bioamplifier (MT11; NEC Medical, Japan) and recorded using a tape recorder (PC216Ax; Sony Precision Technology, Japan).

In this experiment, EGGs were obtained with electrodes arranged for monopolar recordings (Vitrode Bs; Nihon Kohden Inc., Tokyo, Japan). A reference electrode was positioned on the midline of the patient's abdomen near the umbilicus. An active electrode should be placed approximately $10 \mathrm{~cm}$ cephalad from the umbilicus and $6 \mathrm{~cm}$ to the patient's left. It is the position closest to the pacemaker of gastrointestinal motility (Fig. 1).

To remove the noise from the time series of the EGG data $\left\{y_{j} \mid j=0,1,2 \cdots, N-1\right\}$ obtained at $1 \mathrm{kHz}$, resampling was performed at $1 \mathrm{~Hz}$. For the analysis, we obtained a resampled time series $\left\{x_{i} \mid i=0,1,2 \cdots,(N / 1000)-1\right\}$ as follows:

$$
x_{0}=\frac{1}{1000} \sum_{j=0}^{999} y_{j}, x_{1}=\frac{1}{1000} \sum_{j=1 \times 1000}^{1999} y_{j}, \ldots, x_{i}=\frac{1}{1000} \sum_{j=i \times 1000}^{i \times 1000+999} y_{j}
$$

The following delay coordinates were used:

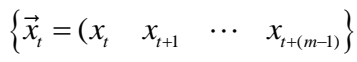

Here, $m$ denotes the embedding dimension. These delay coordinates could be used to reconstruct a continuous trajectory without intersections in an embedding space having a large $m$. The embedding delay, $\tau$, is defined as the minimum delay ( $\tau \geq 0$ ) when the auto-correlation coefficient is zero. In this study, we assumed that there was no connection when the auto-correlation function initially decreased to a value below $1 / e(t \geq 0)$.

\section{B. Calculation Procedure}

In this study, we numerically solved Eq. (1) and Eq. (2) and verified the SR in the stochastic differential equations (SDEs). We converted Eq. (1) and Eq. (2) into difference equations and calculated the numerical solutions using the Runge-Kutta-Gill formula. The initial values were set to $(0$, $0.5)$. Pseudorandom numbers were substituted for $w_{i}(t)(i=1,2)$. These pseudorandom numbers were generated by using the Mersenne twister [10]. These numerical calculations were performed for $N=24000$ time steps. Each time step was 0.05 units.

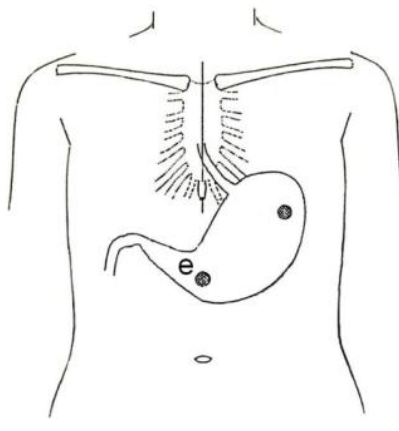

Fig. 1. Positions of electrodes.

The values of the numerical solutions were recorded after every 20 time steps, which is equivalent to a signal sampling rate of $1 \mathrm{~Hz}$. For each value of $\mu$, we obtained 20 numerical solutions of Equations (1) and (2).

1) Using the Wayland and Rosenstein algorithms, estimate the translation errors $\left(E_{\text {trans }}\right)$ and $\operatorname{MLEs}(\lambda)$ in the attractors generating EGG data, except for $30 \mathrm{~min}$ after the postural change. Then, project the stationary EGG onto the $E_{\text {trans }}-\lambda$ plane.

2) Calculate the mean values ( $m(i))$ of $E_{\text {trans }}$ and $\lambda$ for all of the projections obtained in Step (1). According to statistical theory, $95.5 \%$ of the EGGs would project onto the region $\left\{\mathfrak{R}_{s}^{2} \mid m\left(E_{\text {trans }}\right) \pm 2 \sigma\left(E_{\text {trans }}\right) m(\lambda) \pm 2 \sigma(\lambda)\right\}$ as shown in Fig. 2.

3) Calculate the standard deviations $(\sigma(i))$ of $E_{\text {trans }}$ and $\lambda$ for all of the projections obtained in Step (1).

4) Project the numerical solutions of Equation (1) onto the $E_{\text {trans }}-\lambda$ plane obtained in Step (1).

5) Count the number of numerical solutions projected onto region $\mathfrak{R}_{s}^{2}$ of the $E_{\text {trans }}-\lambda$ plane.

6) Calculate the conformity ratio of the number counted in Step (5) to 20, i.e., the number of numerical solutions for each value of $\mu$.

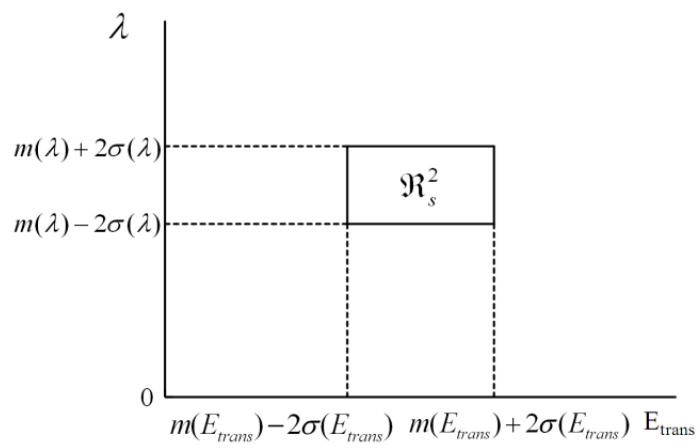

Fig. 2. Region $\mathfrak{R}_{s}^{2}$. 


\section{RESULTS}

\section{A. Subjective Evaluation}

We analyzed the EGG data. The Wayland and Rosenstein algorithms were applied to the attractors in the case of all 42 (14 subjects $\times 3=42$ EGGs) EGG data items.

The attractors of the EGGs were reconstructed in accordance with Takens' embedding method. The form of the attractors could be evaluated by $E_{\text {trans }}$ and $\lambda$. The embedding delays and embedding dimensions were distributed from 2 (s) to 4 (s) and from 2 to 7 , respectively.

The translation errors were distributed from 0.31 to 0.51 . The average \pm standard deviation of $E_{\text {trans }}$ was found to be $0.42 \pm 0.044$.

The MLEs were distributed from 0.67 to 0.78 . All of the MLEs were greater than 0 . The average \pm standard deviation of the MLEs derived from the EGG data was found to be 0.72 \pm 0.026 .

\section{B. Simulation Evaluation}

In the 24000 time steps, there was no incidence where the numerical solutions did not diverge for $\mu=1,2, \cdots, 20$; the value of $\tau$ derived from the first component of the numerical solution was similar to that derived from the second component. We compared this numerical solution with the EGG data.

The cross-correlation coefficient between the observed signal $x(t)$ and the periodic function $s(t)$ was calculated as a substitute for the signal-to-noise ratio (SNR) used in previous studies, in which the occurrence of the SR was investigated. The cross-correlation coefficient between the numerical solutions, $\dot{x}$, and the periodic function, $s(t)$, in Eq. (1) was maximized for a moderate value of noise intensity, $\mu=12$. Thus, the SR could be generated using Eq. (1) and Eq. (2) with $\mu=12$. Numerical solutions were projected onto the $E_{\text {trans }}-\lambda$ plane (Fig. 3).

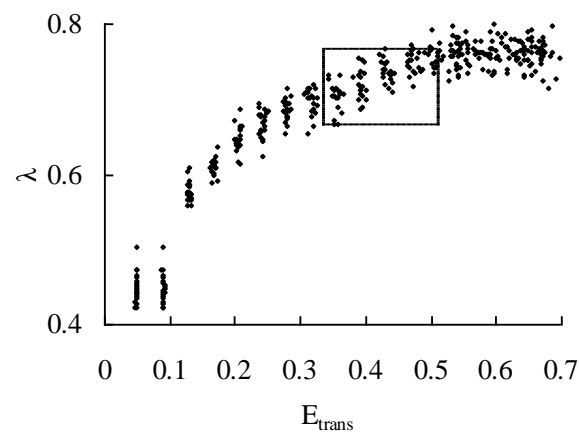

Fig. 3. $E_{\text {trans }}-\lambda$ plane (simulation results).

\section{DISCUSSION}

In this study, we analyzed EGG time-series data using complex dynamical methods. $E_{\text {trans }}$ and $\lambda$ were calculated from the EGG data.

SDEs were proposed as a mathematical model of an EGG by Matsuura et al. [4]. SR occurs for an appropriate coefficient, $\mu$. Some biosystems are based on the nonlinear phenomenon of SR, in which the detection of small afferent signals can be enhanced by the addition of an appropriate amount of noise [10]. We examined whether or not the SR generated using Eq. (1) and Eq. (2) could describe an EGG time series. The EGGs are well described by the SDEs in the case of $\mu=10$, which might not represent the SR. We therefore claim that the SR is not legitimate as a mathematical model of the EGG in the elderly but that the SR remains statistically applicable to the mathematical model of the stationary EGG in the healthy young [11].

The periodic function and noise in Eq. (1) and Eq. (2) represent the intestinal movements and other biosignals, respectively. The numerical solutions associated with the stochastic resonance are strongly correlated with the periodic function $(s(t))$, where the periodic function represents the intestinal movements $(6 \mathrm{cpm})$. Conversely, there are synergies in the activity owing to parasympathetic nerve control in accordance with physiology [12]. This synergy influences the electric activity of the stomach and intestinal motility. The physiological meaning of the SR as a mathematical model of an EGG was considered. The gastric electrical activity in an elderly person might not synchronize with the intestinal activity.

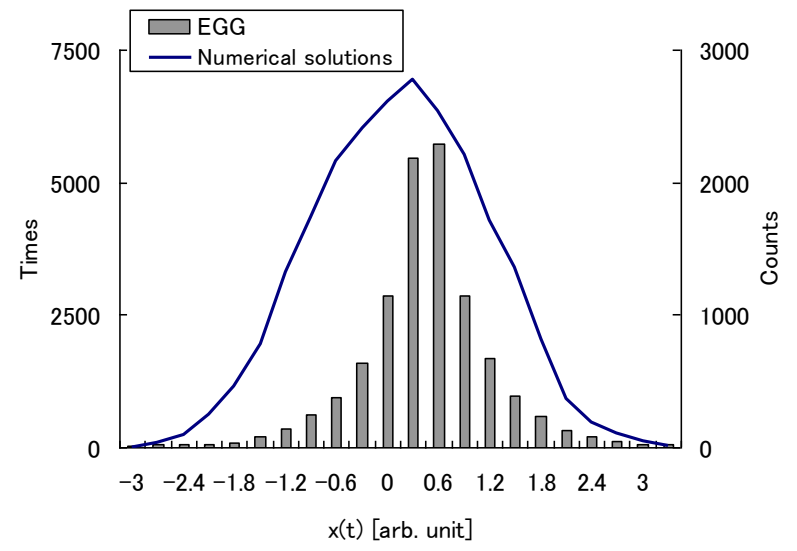

Fig. 4. Distributions of EGGs and numerical solutions $(\mu=10)$.

According to the chi-square test, distribution of the numerical solutions in the SR does not fit the distribution of the EGGs in the elderly, as shown in Fig. 4 ( $p<0.05)$. Based on this distribution of the EGG, the form of the potential function, $f(x)$, in Eq. (1) should be discussed in the next step.

The diseases resulting from constipation and erosive gastritis (an illness in which the inside of the stomach becomes swollen and painful) are accompanied by anomalous autonomic nervous activity. A decline in the electrical activity of the stomach would likely change the degree of determinism $\left(E_{\text {trans }}\right)$ and the complexity $(\lambda)$ in the attractor reconstructed from the EGG data. With the addition of the mathematical model of an EGG, electrogastrography will be instrumental in the diagnosis of diseases of the alimentary canal and autonomic nervous system.

\section{CONCLUSION}

In our development of a mathematical model of an EGG, we added the Van der Pol equation to a periodic function and random white noise to represent the intestinal motility and other biosignals. By projecting the data from a stationary EGG obtained for a subject in the supine position, along with 
the numerical solutions, onto the $E_{\text {trans }}-\lambda$ plane, we were able to qualitatively evaluate the affinity between them. We succeeded in discovering that the SR remains statistically applicable to the mathematical model of the stationary EGG in the healthy young but that the SR could not be accurately applied as a mathematical model of the EGG in the elderly. To increase the reliability of this mathematical model method, it would be necessary to perform further investigations by increasing the number of EGGs studied.

\section{REFERENCES}

[1] W. C. Alvarez, "The electrogastrogram and what it shows," Journal of the American Medical Association, vol. 78, pp. 1116-1118, 1922.

[2] D. Couturier, C. Roze, J. Paolaggi, and C. Debray, "Electrical activity of the normal human stomach. A comparative study of recordings obtained from the serosal and mucosal sides," Digestive Diseases and Sciences, vol. 17, pp. 969-976, 1972.

[3] L. K. Kenneth and M. Robert, Handbook of Electrogastrography, U.K.: Oxford University Press, 2004.

[4] Y. Matsuura, H. Takada, K. Yokoyama, and K. Shimada, "Proposal for a new diagram to evaluate the form of the attractor reconstructed from electrogastrography," Forma, vol. 23, no. 1, pp. 25-30, 2008.

[5] R. Wayland, D. Bromley, D. Pickett, and A. Passamante, "Recognizing determinism in a time series," Physical Review Letters, vol. 70, pp. 580-582, 1993.

[6] F. Takens, "Detecting strange attractors in turbulence," Lecture Notes in Mathematics, vol. 898, pp. 366-381, 1981

[7] S. Sato, M. Sano, and Y. Sawada, "Practical methods of measuring the generalized dimension and the largest Lyapunov exponent in high dimensional chaotic systems," Progress of Theoretical Physics, vol. 77, pp. 1-5, 1987.

[8] M. T. Rosenstein, J. J. Collins, and C. J. De Luca, "A practical method for calculating largest Lyapunov exponents from small data series," Physica D, vol. 65, pp. 117-134, 1993.

[9] R. Benzi, A. Sutera, and A. Vulpiani, "The mechanism of stochastic resonance," Journal of Physics A, vol. 14, pp. 453-457, 1981.

[10] M. Matsumoto and T. Nishimura, "A 623-dimensionally equidistributed uniform pseudorandom number generator," $A C M$ Transaction Modeling and Computer Simulation, vol. 8, no. 1, pp. 3-30, 1998.

[11] Y. Matsuura, M. Miyao, and H. Takada, "Stochastic resonance as a mathematical model of an electrogastrogram," Journal of Physical Science and Application, vol. 2, no. 6, pp. 186-194, 2012.

[12] R. M. Berne and M. N. Levy, Physiology, 4th ed. St. Louis, U.S.A.: Mosby-Year Book, 1998

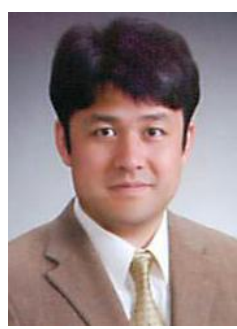

Hiroki Takada was born in July 1973 at Kuwana, Japan. In 2002, he graduated from Graduate School of Mathematics, Nagoya University where he completed his under-graduate and post-graduate study. He received doctorate by way of dissertation (DSc) in 2004. During 2002-2006, he worked for Nagoya University as a postdoctoral research fellow in several fields such as physics, environmental medicine, and computational science. Since 2006, he became an associate professor. $\mathrm{He}$ worked for
Department of Radiology, Gifu University of Medical Science. In the recent personal changes, he transferred to Department of human \& artificial intelligent systems, University of Fukui. He is currently at Graduate School of Engineering, University of Fukui and a guest researcher for Aichi Medical University School of Medicine. Now he has more than 80 publications such as Electromyography: New Developments, Procedures and Applications (NOVA Publisher: New York, 2012), Depth Map and 3D Imaging Applications: Algorithms and Technologies (Chapter 15) (IGI global: Hershey, PA, USA, 2011) and Frontiers of Computational Science (Chapter 57) (Springer-Verlag: Berlin, 2005). Broadly, his research lies in Mathematical Physics (especially, Stochastic Process Theory). Applying this theoretical background, the author enjoys doing research in Environmental Physiology for preventive medicine. He is also interested in nonlinear analysis of biosignal such as electrocardiogram, continuous blood pressure, cerebral blood flow, micro-neurogram, stabilogram, electromyogram, skin potential, and electrogastrogram. His research is funded by Grant-in-Aid for Science Research in Japan. The author received many awards including an award for encouragement from Society for Science on Form in 2002. He serves as an executive editor of "Journal of Sports Medicine and Doping Study (USA)". He is also an associate editor in chief of "FORMA", a member of IEEE and Program Committee of "International Conference on Bio-inspired systems and signal processing".

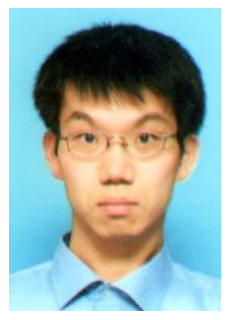

Yasuyuki Matsuura was born at Nagoya, Japan. The author received $\mathrm{Ph} . \mathrm{D}$. degree in science from Nagoya City University (Nagoya, Japan) in 2009. During 2009-2010, he worked Researcher in Department of Hygiene, Graduate School of Medicine, Nagoya City University. During 2010-2011, he worked for Nagoya University as a postdoctoral research fellow in several fields such as physics, public health, and computational science. He is currently a postdoctoral research fellow at Headquarters for Innovative Society-Academia Cooperation, University of Fukui, Japan since 2011. Now he has more than 30 publications such as Electromyography: New Developments, Procedures and Applications (Chapter 8) (NOVA Publisher: New York, 2012), Depth Map and 3D Imaging Applications: Algorithms and Technologies (Chapter 15) (IGI global: Hershey, PA, USA, 2011) and Frontiers of Computational Science (Chapter 57) (Springer-Verlag: Berlin, 2005). Broadly, my research lies in Medical Engineering (especially, Biological Signal Processing). Applying this theoretical background, the author enjoys doing research in Environmental Physiology for preventive medicine. He is also interested in the development of signal processing algorithms for analysis of biological systems. He is currently focusing his studies on computational modeling of electrogastrogram, and on application of non-linear analysis methods to characterize biological signals (Electrocardiogram, Electrogastrogram, Stabilogram, et al.). He is a member of IEEE and the Engineering in Medicine and Biology Society. 